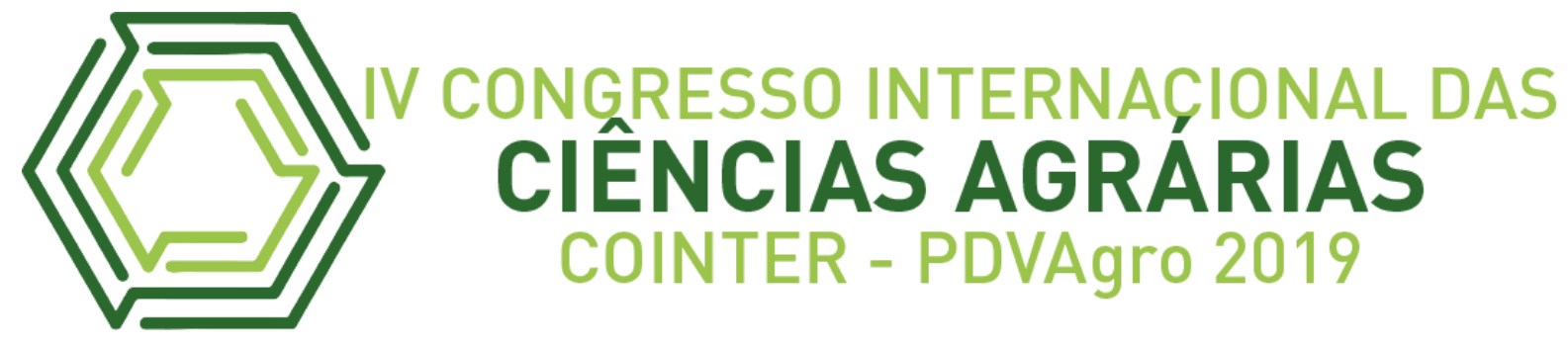

\title{
A IMPORTÂNCIA COMERCIAL E SUSTENTÁVEL DA MAÇARANDUBA (Manilkara huberi - Ducke)
}

\section{LA IMPORTANCIA COMERCIAL Y SOSTENIBLE DE MAÇARANDUBA (Manilkara huberi - Ducke)}

\section{THE COMMERCIAL AND SUSTAINABLE IMPORTANCE OF MAÇARANDUBA (Manilkara huberi - Ducke)}

Apresentação: Comunicação Oral

Mariele Correia Alves ${ }^{1}$; Camila Nunes Sagais ${ }^{2}$; Hellem Dayane dos Santos Pinheiro ${ }^{3}$;Klewton Adriano Oliveira Pinheiro ${ }^{4}$; Sanae Nogueira Hayashi ${ }^{5}$

DOI: https://doi.org/10.31692/2526-7701.IVCOINTERPDVAgro.2019.0096

\section{Resumo}

A Maçaranduba (Manilkara huberi - Ducke) da família Sapotaceae é uma espécie frequente em vários estados do Brasil e muito procurada por ser de grande porte, ter resistência a pragas e possuir uma madeira densa e economicamente viável. Conhecer o crescimento das espécies de um povoamento florestal, principalmente daquelas de interesse econômico, é de fundamental importância para o manejo florestal, visando compreender esse processo, o presente estudo buscou avaliar como a espécie se comporta depois da exploração florestal e tratamento silvicultural e conhecer a intensidade de corte que possibilite a sustentabilidade da espécie em uma floresta manejada sob um regime de um sistema policíclico. Nesse sentido, avaliou-se o processo dinâmico do crescimento da população de maçaranduba, no período de 1984-2011, em uma área de 40 hectares localizada no município de Vitoria do Jari no estado do Amapá. Adotouse a metodologia de instalação de parcelas permanentes para inventário florestal contínuo. Cada parcela de 1 ha foi dividida em 100 subparcelas de 10m x 10m, para facilitar as atividades de monitoramento. A identificação dos indivíduos foi realizada, na floresta, pelo nome vulgar por técnicos experientes da Embrapa Amazônia Oriental. A maçaranduba é uma das espécies mais

\footnotetext{
${ }^{1}$ Graduação em Agronomia, Universidade Federal Rural da Amazônia, marielealves114@gmail.com

${ }^{2}$ Graduação em Agronomia, Universidade Federal Rural da Amazônia, sagais.camila@gmail.com

${ }^{3}$ Graduação em Agronomia, Universidade Federal Rural da Amazônia, danypinheiro.d@gmail.com

${ }^{4}$ Doutorado, Instituto Federal de Educação, Ciência e Tecnologia do Pará, klewton.pinheiro@gmail.com

${ }^{5}$ Doutorado, Universidade Federal Rural da Amazônia, sanae.hayashi@ufra.edu.br
} 


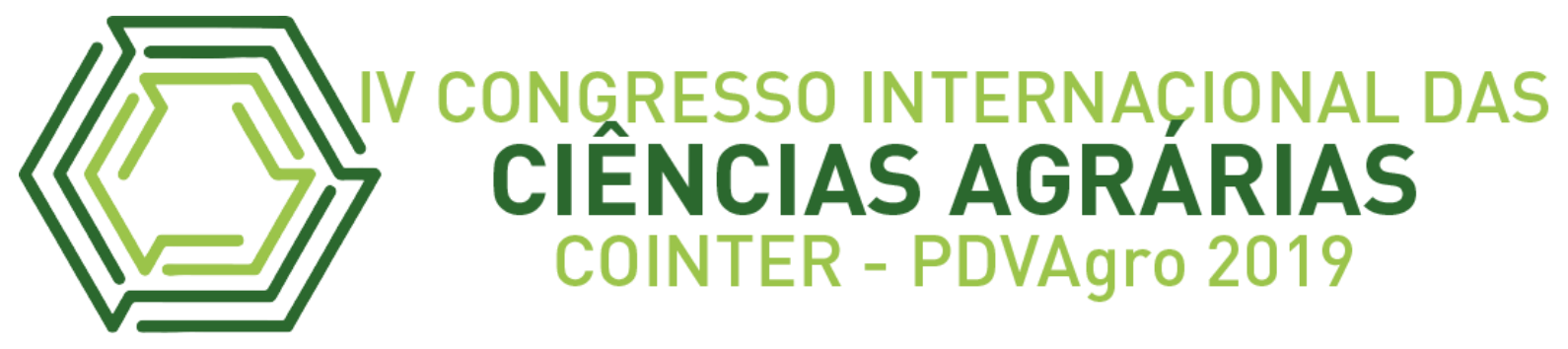

abundantes da área estudada (36 hectares) registrada com 2.515 indivíduos e um volume total de $327,337 \mathrm{~m}^{3}$. Devido ao efeito da abertura do dossel provocar diminuição na competição por espaço e luz no crescimento das árvores, influenciou na redução do crescimento populacional da Maçaranduba.

Palavras-Chave: Silvicultural, sustentabilidade, manejo.

\section{Resumen}

La Macaranduba (Manilkara huberi - Ducke) de la familia Sapotaceae es una especie común en muchos estados de Brasil y es muy popular porque es grande, tiene resistencia a las plagas y tiene una madera densa y económicamente viable. Conocer el crecimiento de las especies de un rodamiento forestal, especialmente las de interés económico, es de importancia fundamental para el manejo forestal, para comprender este proceso, el presente estudio buscó evaluar cómo se comporta la especie después de la tala y tratamiento silvícola y conocer la intensidad de corte que permite la sostenibilidad de la especie en un bosque manejado bajo un régimen de un sistema policíclico. En este sentido, evaluamos el proceso dinámico del crecimiento de la población de maçaranduba (Manilkara huberi - Ducke), de 1984 a 2011, en un área de 40 hectáreas ubicada en el municipio de Vitoria do Jari en el estado de Amapá. Se adoptó la metodología de instalación de parcelas permanentes para inventario forestal continuo. Cada parcela de 1 ha se dividió en 100 subparcelas de 10m x 10m para facilitar las actividades de monitoreo. La identificación de individuos se realizó en el bosque con el nombre común de técnicos experimentados de Embrapa Amazonía Oriental. Maçaranduba es una de las especies más abundantes en el área de estudio (36 hectáreas) registrada con 2,515 individuos y un volumen total de $327,337 \mathrm{~m}^{3}$. Debido al efecto de la apertura del dosel que causa una disminución en la competencia por el espacio y la luz en el crecimiento de los árboles, influyó en la reducción del crecimiento de la población de Maçaranduba.

Palabras Clave: Silvicultura, sostenibilidad, manejo.

\section{Abstract}




\section{CIENCIAS AGRARIAS
COINTER - PDVAGO 2019}

The Maçaranduba (Manilkara huberi - Ducke) of the Sapotaceae family is a common species in many states of Brazil and is very popular because it is large, has pest resistance and has a dense and economically viable wood. Knowing the growth of species in a forest stand, especially those of economic interest, is of fundamental importance for forest management, in order to understand this process, the present study sought to evaluate how the species behaves after logging and forestry treatment and to know the intensity of cut that allows the sustainability of the species in a forest managed under a regime of a polycyclic system. In this sense, we evaluated the dynamic process of population growth of maçaranduba), from 1984-2011, in an area of 40 hectares located in the municipality of Vitoria do Jari in the state of Amapá. It was adopted the methodology of installation of permanent plots for continuous. Each 1 ha plot was divided into $10010 \mathrm{~m} \mathrm{x} \mathrm{10m} \mathrm{subplots} \mathrm{to}$ facilitate monitoring activities. The identification of individuals was performed in the forest by the common name by experienced technicians from Embrapa Eastern Amazon. Maçaranduba is one of the most abundant species in the study area (36 hectares) recorded with 2,515 individuals and a total volume of $327,337 \mathrm{~m}^{3}$. Due to the effect of canopy opening causing a decrease in competition for space and light in tree growth, it influenced the reduction in population growth of Maçaranduba.

Keywords: Forestry, sustainability, management.

\section{Introdução}

A Amazônia apresenta a maior reserva de recursos florestais e uma das maiores biodiversidades do Planeta, porém, seus recursos naturais (madeireiros e não madeireiros) estão sendo explorados de forma irracional, causando danos ao ecossistema como um todo (FRANCEZ, 2009). O setor madeireiro contribui de forma expressiva com a economia regional e nacional e, também, para a geração de emprego e o bem-estar social (SABOGAL, 2006). Há uma grande procura por espécies consideradas "nobres", devido sua qualidade e resistência a pragas comuns em madeira.

Segundo Loureiro (1979) e Superintendência de Desenvolvimento da Amazônia-SUDAM (1979), a Maçaranduba da família Sapotaceae espécie frequente 


\section{CONGRESSO INTERNACIONAL DAS CIENCIAS AGRARIAS COINTER - PDVAgro 2019}

em vários estados do Brasil, se torna muito procurada devido as suas características peculiares, como seu porte, que pode chegar até 50 metros, a grande densidade de sua madeira, sua resistência a pragas e também por ser uma espécie não muito cara em comparação com outras espécies.

Segundo Souza et al., (2002), sua distribuição geográfica é generalizada principalmente na região Amazônica, em zonas de terra firme, planalto e flanco. Segundo Loureiro e Silva (1968), sua concentração ocorre principalmente nos estados da Região Norte, mais frequente no Pará, Roraima, Amazonas, Amapá, Rondônia e nordeste do Maranhão.

De acordo com Gayot; Sist. (2004), num estudo de exploração de impacto florestal nas populações de Manilkara Huberi, M. Bidendata, e M. Paraenses, a espécie Manilkara Huberi apresentou taxas de regeneração natural mais baixa que as demais estudadas, concluindo-se que numa exploração seletiva, baseada em um diâmetro mínimo de exploração de $55 \mathrm{~cm}$, e considerando um ciclo de corte de 30 anos, não será possível manter a sustentabilidade da população da espécie com densidade economicamente viável. Isso sugere que a rotação dessa espécie em florestas naturais poderá ser longa.

A maçaranduba é uma das espécies mais abundantes da área estudada com 2,515 indivíduos, e também uma das espécies mais procuradas no mercado por sua qualidade. Segundo a Associação das Indústrias Exportadoras de Madeira do Estado do Pará- AIMEX (2005) e Schulze et al., (2005), a maçaranduba está entre as principais espécies florestais exportadas pelo estado do Pará, na forma de madeira serrada, com mercado seguro nos Estados Unidos da América, Japão, e vários países europeus.

O objetivo desse levantamento acerca da importância comercial sustentável da Maçaranduba é identificar como a espécie se comporta depois da exploração e conhecer a intensidade de corte que possibilite a sustentabilidade da espécie em uma floresta manejada sob um regime de um sistema policíclico. 


\section{UIV CONGRESSO INTERNACIONAL DAS CIEENCIAS AGRARIAS COINTER - PDVAgro 2019}

\section{Fundamentação Teórica}

O conhecimento da fitossociologia e dinâmica das florestas tropicais torna-se um elemento essencial no momento de escolher o melhor sistema silvicultural para regenerar a floresta. Segundo Jardim e Hosokawa (1987), é importante que se conheça a estrutura de florestas equatoriais como as da Amazônia, para que se possa aplicar um sistema de manejo com base no rendimento sustentável.

A base para se ter conhecimento da dinâmica ecológica são as informações obtidas pelas análises estruturais da floresta e de cada população de espécies, tanto antes da exploração florestal como em longos períodos após essa atividade, pois, segundo Carvalho (2001), a recuperação da estrutura da floresta após a colheita de madeira é lenta, principalmente quando é submetida a altos níveis de exploração com diferentes tipos de manejo.

As espécies que precisam ter suas populações estudadas em termos dinâmicos, tanto em relação à ecologia como à economia, são principalmente aquelas com valor de mercado agregado e que por isso, têm suas populações mais alteradas. Portanto, decidiu-se estudar a estrutura da população da Manilkara huberi (Ducke), uma das espécies que tem a sua madeira mais colhida na Amazônia e comercializada no mercado nacional e internacional.

A maçaranduba é bastante valorizada no mercado, por possuir madeira muito pesada, dura e resistente, com densidade básica de 0,87 g cm-3. Essas características contribuem para que a espécie seja uma das mais exploradas na região amazônica, por isso torna-se imprescindível conhecer o seu comportamento para possibilitar a aplicação de um manejo adequado e, assim, garantir a sua conservação na estrutura florestal.

\section{Metodologia}

Características e localização da área de estudo 


\section{CONGRESSO INTERNACIONAL DAS CIEENCIAS AGRARIAS COINTER - PDVAgro 2019}

A floresta em estudo está localizada no município de Vitoria do Jari no estado do Amapá (Figura 1), entre as coordenadas 5²0’20" W e $0^{\circ} 00^{\prime} 55^{\prime \prime} \mathrm{S}$, a uma altitude de aproximadamente $150 \mathrm{~m}$. O clima nessa região é do tipo Ami, pela classificação de Köppen (AZEVEDO et al., 2008).

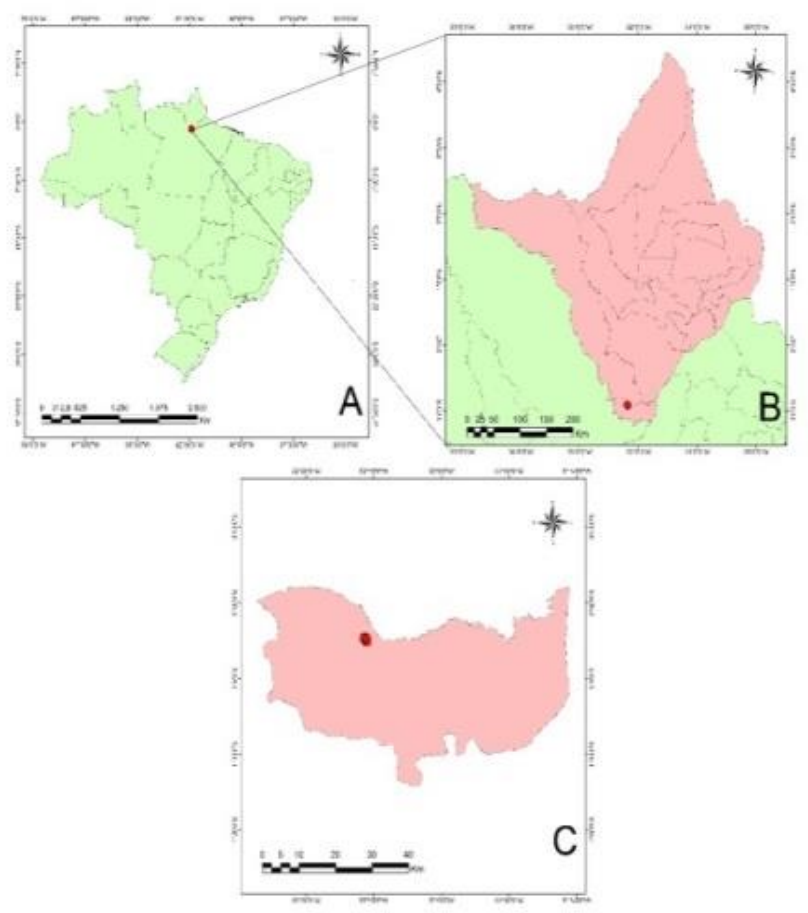

Figura 1. Localização das parcelas de monitoramento permanentes da empresa Jari, na localidade Morro do Felipe (C), Vitória do Jari, (B) Estado do Amapá, Brasil (A)

A precipitação média anual alcança $2.234 \mathrm{~mm}$, com um período chuvoso de dezembro a maio. Ocorre uma estação seca de três meses, que se inicia em junho e que se caracteriza por uma precipitação mensal inferior a $8 \%$ do volume anual de chuva. A temperatura média anual é de $25,80^{\circ} \mathrm{C}$, com a amplitude térmica variando mais ou menos $20^{\circ} \mathrm{C}$ entre o máximo e o mínimo valor mensal.

Na Jari a vegetação é do tipo Floresta Ombrófila Densa. Os solos são do tipo Latossolo Amarelo Distrófico, com textura argilosa pesada (AZEVEDO et al., 2008). O experimento foi iniciado em 1983, com a realização do inventário pré-exploratório considerando todas as árvores com DAP $\geq 50 \mathrm{~cm}$. Em 1985 foi realizada a exploração 


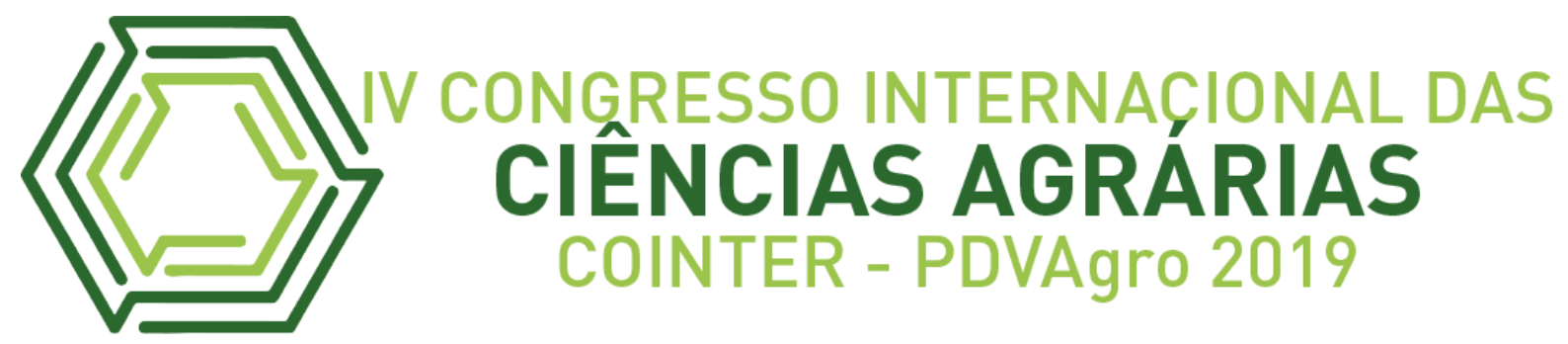

florestal na área de floresta primária, retirando $15 \%, 25 \%$ e $35 \%$ do volume total das árvores com DAP $\geq 50 \mathrm{~cm}$, correspondendo, aproximadamente, a $25 \mathrm{~m}^{3} / \mathrm{ha}, 40 \mathrm{~m} / \mathrm{ha}$ e $60 \mathrm{~m}^{3} / \mathrm{ha}$, respectivamente.

Foram exploradas árvores com DAP $\geq 60 \mathrm{~cm}$, de 42 espécies de valor comercial na região. O tratamento silvicultural foi realizado em 1994, aplicando-se dois tipos de desbaste: desbaste sistemático, com duas intensidades de redução da área basal original (30\% e $50 \%)$ e o desbaste seletivo.

\section{Metodologia e coleta de dados}

Adotou-se a metodologia de instalação de parcelas permanentes para inventário florestal contínuo sugerida por Silva; Lopes (1984), revisada e atualizada por Silva et al., (2005). Cada parcela de 1 ha foi dividida em 100 subparcelas de $10 \mathrm{~m}$ $\mathrm{x} 10 \mathrm{~m}$, para facilitar as atividades de monitoramento. As parcelas e subparcelas foram demarcadas com piquetes, os quais foram pintados na parte superior com tinta vermelha à base de óleo para diferenciá-los da vegetação.

Os procedimentos para coleta e registro dos dados nas parcelas permanentes foram àqueles estabelecidos nas diretrizes propostas por (SILVA et al, 2005). Foram realizadas sete medições, a cada 2 anos, durantes 27 anos: a primeira foi em 1980 e a ultima em 2010.

A identificação dos indivíduos foi realizada, na floresta, pelo nome vulgar por técnicos experientes da Embrapa Amazônia Oriental. Foi coletada material botânica das espécies menos comuns e dos grupos de espécies que suscitaram dúvidas, para ser identificado, através de comparação, no Herbário IAN da Embrapa Amazônia Oriental.

Procedimento Laboratorial e cálculos

Para o processamento dos dados foram utilizados os programas: MFT (Monitoramento de Florestas Tropicais), desenvolvido pela Embrapa Amazônia Oriental, que neste trabalho servirá para analisar os parâmetros referentes à florística e à estrutura da floresta.

Sobre o cálculo da composição florística: os números de espécies, gêneros e famílias foram determinados, considerando todos os indivíduos a partir de $5 \mathrm{~cm}$ de 


\section{UIn CONGRESSO INTERNAGIONAL DAS CIEENCIAS AGRARIAS COINTER - PDVAgro 2019}

diâmetro. Foi elaborada uma lista de espécies existentes na área, com os nomes locais, nomes científicos e famílias.

Índice de similaridade de Sorensen, através da seguinte equação 1

$$
C s=\frac{2 j}{(a+b)} \quad \text { (Equação 1) }
$$

No qual: Cs= Índice de Sorensen; $\mathrm{j}=$ Número de espécies comuns entre a e b; $a=$ Número de espécies da comunidade $a ; b=$ Número de espécies da comunidade $b$.

Sobre o cálculo da diversidade florística: foi determinada a diversidade de espécies comparando-se a floresta manejada com a não perturbada assim como para os diferentes tratamentos e entre as varias medições realizadas. Sobre a riqueza e equabilidade: a diversidade de espécies e a equabilidade de espécies foram determinadas através do Índice de Shannon, que vem sendo largamente utilizado em florestas amazônicas (ex: BARROS et al., 2000; MIRANDA, 2000). A diversidade é obtida pela equação 2 :

$$
H^{\prime}=-\sum_{i}^{n} \text { pi Ln } \quad \quad \text { (Equação 2) }
$$

Sendo: H': Índice de Shannon-Wiener; pi: proporção de indivíduos de uma determinada espécie na área; Ln: Logarítmo neperiano; pi = ni / N; ni: número de indivíduos da espécie i; e N: número total de indivíduos na área de estudo.

A equabilidade é obtida através da equação 3:

$$
J=\frac{H^{\prime}}{\operatorname{Ln} S}
$$

No qual: J: Índice de equabilidade; H': Índice de Shannon-Wiener; S: número total de espécies; e Ln: Logarítmoneperiano. 


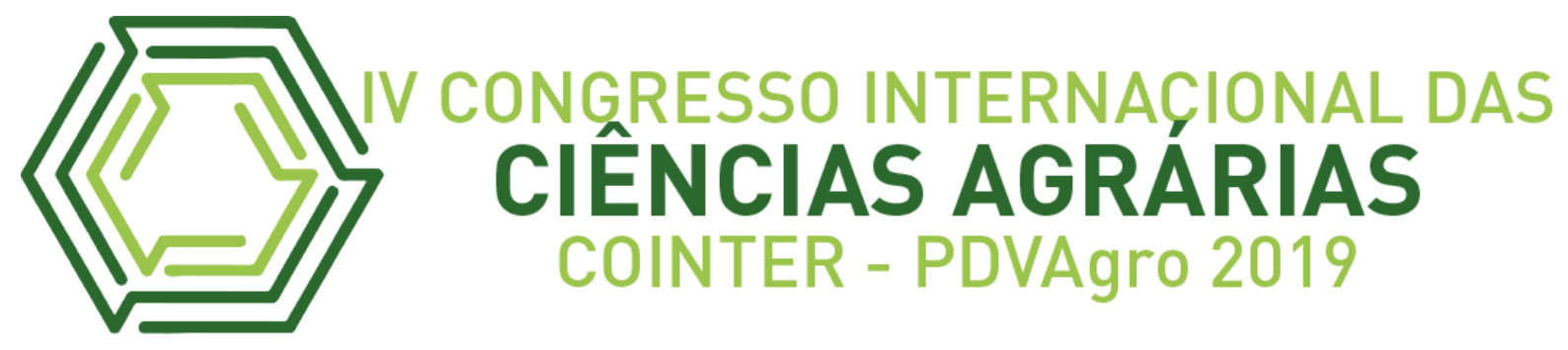

A estrutura diamétrica da floresta foi calculada através da abundância por classe diamétrica, e foram determinada o estoque de volume entre florestas manejadas e não perturbadas assim como para os diferentes tratamentos e entre as várias medições realizadas, através de equações de volumes proposta por Silva; Araújo (1984) e Silva et al., (1984).

O delineamento experimental das áreas estudadas foi o de blocos ao acaso com três repetições. As alternativas de manejo testadas foram combinações de intensidades de exploração com intensidades de desbastes. As intensidades de exploração (15\% intensidade leve, 25\% - intensidade média e 35\% - intensidade pesada) foram representadas por porcentagens do volume extraído em relação ao volume total da floresta, a partir do diâmetro à altura do peito (DAP) de $60 \mathrm{~cm}$. Foram combinadas com quatro tipos de intensidades de desbastes, o desbaste sistemático e o desbaste seletivo nas intensidades de 0\%,30\%, 50\% e 70\% de redução da área basal original.

\section{Resultados e Discussão}

Ao todo foram amostradas 12 árvores adultas em testemunha e 306 árvores em tratamento, totalizando 40 parcelas, divididas em testemunha com 4 parcelas de 1 ha cada e tratamento com 36 parcelas de 1 ha cada, o que resultou em uma densidade final de 3 adultos.ha ${ }^{-1}$ e de 8,5 adultos.ha ${ }^{-1}$, respectivamente. Considerando toda a área amostrada (40 ha) a densidade média de maçaranduba foi de 8,0 adultos.ha ${ }^{-1}$. Nenhum indivíduo jovem foi amostrado.

Tabela 1. Valores correspondentes de abundância (ind.), volume $\left(\mathrm{m}^{3} / \mathrm{ha}\right)$ e área basal ( $\mathrm{G} \mathrm{m}^{2} / \mathrm{ha}$ ) por ano da espécie Manilkara huberi (Ducke), em uma área de 4 ha de floresta nativa da Jari, Amapá, Brasil. 


\section{UIn CONGEESO INTERACLIONAL DAS CIENCIAS AGRARIAS COINTER - PDVAgro 2019}

\begin{tabular}{llll}
\hline \multirow{2}{*}{ Ano } & \multicolumn{2}{l}{ Testemunha } & \\
\cline { 2 - 4 } & Ind. & $\mathrm{G} \mathrm{m}^{2} / \mathrm{ha}$ & Volume $\mathrm{m}^{3} / \mathrm{ha}$ \\
\hline 1984 & 6 & 1,632 & 1,532 \\
1986 & 6 & 1,664 & 1,542 \\
1988 & 5 & 1,357 & 1,265 \\
1990 & 6 & 1,738 & 1,569 \\
1994 & 7 & 1,837 & 1,764 \\
1996 & 7 & 1,869 & 1,775 \\
2004 & 12 & 2,208 & 2,731 \\
2011 & 12 & 2,408 & 2,826 \\
\hline
\end{tabular}

Observamos na Tabela 1 que no ano de 1988 foi o ano onde se teve o menor número de indivíduos, já nos anos de 2004 e 2011 foram os anos onde se teve o maior número de indivíduos consequentemente a maior volumetria. Houve uma constância entre os anos de 1984 e 1996 com aproximadamente a mesma quantidade de indivíduos. Neste período a maçaranduba acumulou $14,713 \mathrm{~m}^{2}$ de área basal e $15,004 \mathrm{~m}^{3}$ de volume.

Oliveira (1995) verificou que, em floresta secundária, as árvores totalmente iluminadas cresceram $30 \%$ mais do que as árvores parcialmente sombreadas e $60 \%$ mais que as árvores totalmente sombreadas, um dos motivos pelo qual a espécie com o passar do tempo apresentou um crescimento populacional significativo até o momento onde se obteve dados dessa espécie na área.

A exploração acelerou as taxas de incremento da espécie, embora, com o passar do tempo este efeito foi se dissipando e, atualmente, as taxas provavelmente tendem a estabilização. A maçarandubra (Manilkara huberi) é uma espécie tolerante à sombra que para acelerar seu crescimento necessita de iluminação.

A área basal em 36 hectares (tratamento) foi de $0,92 \mathrm{~m}^{2} \mathrm{ha}^{-1}$ considerando os dados de inventário $100 \%$. Esse resultado difere bastante de um estudo feito em 84 ha 


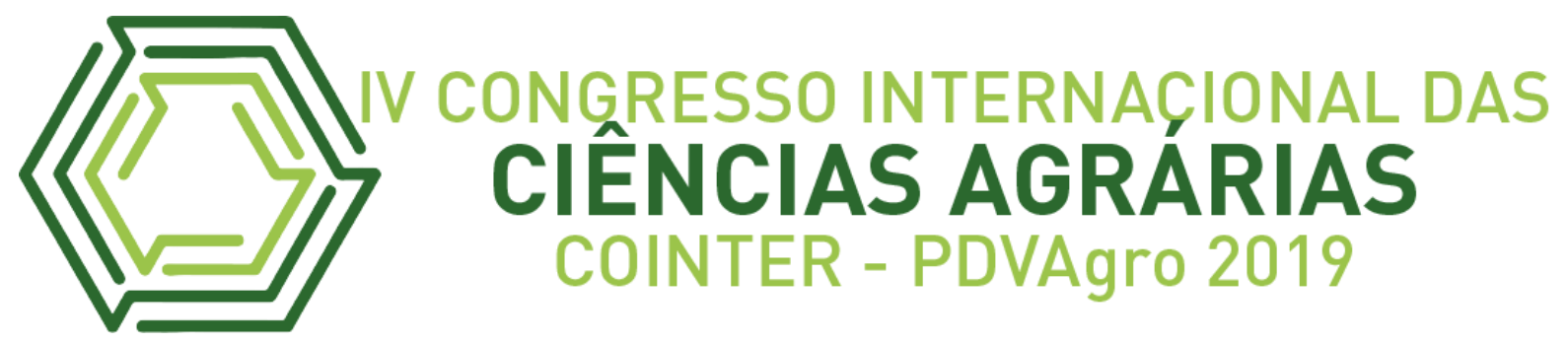

de floresta natural na Fazenda Rio Capim em Paragominas onde foram encontrados apenas 0,32 $\mathrm{m}^{2} \mathrm{ha}^{-1}$ (PINHEIRO, 2008). O volume total da espécie na área de 36 hectares foi de $327,337 \mathrm{~m}^{3}$ o que daria uma média de $9,092 \mathrm{~m}^{3} \mathrm{ha}^{-1}$ (Tabela 2).

Tabela 2: Valores correspondentes de abundância (ind.), volume $\left(\mathrm{m}^{3} / \mathrm{ha}\right)$ e área basal ( $\mathrm{G} \mathrm{m}^{2} / \mathrm{ha}$ ) por ano da espécie Manilkara huberi (Ducke), em uma área de 36 ha de floresta nativa da Jari, Amapá, Brasil.

\section{Tratamento}

\begin{tabular}{cccc}
\cline { 2 - 4 } Ano & Ind. & $\mathrm{G} \mathrm{m}^{2} / \mathrm{ha}$ & \multicolumn{2}{c}{ Volume } \\
& & & $\mathrm{m}^{3} / \mathrm{ha}$ \\
\hline 1984 & 412 & 63,069 & 57,196 \\
1986 & 302 & 33,899 & 40,114 \\
1988 & 299 & 34,151 & 39,443 \\
1990 & 300 & 34,202 & 39,082 \\
1994 & 297 & 34,583 & 38,649 \\
1996 & 302 & 35,585 & 39,067 \\
2004 & 297 & 34,183 & 37,595 \\
2011 & 306 & 33,259 & 36,191 \\
\hline
\end{tabular}

Observamos na Tabela 2 que a população de maçaranduba entre anos de 1984 e 2011 a espécie se manteve em equilíbrio quanto ao número de indivíduos, houve uma pequena taxa de mortalidade da espécie, mas não foram resultados alarmantes é importante salientar que o crescimento lento na fase de plântulas, fase muito apropriada aos predadores expõe essa espécie a uma alta mortalidade no início da vida, portanto, devemos planejar de forma sustentável a sua exploração e mantê-la para futuras gerações.

Sendo assim, as taxas apresentadas para a maçaranduba na área, principalmente após o efeito da exploração, são coerentes com taxas de crescimento de espécies tolerantes à sombra em florestas tropicais. No entanto, a comparação das taxas de 


\section{CONGRESSO INTERNACIONAL DAS CIENCIAS AGRARIAS COINTER - PDVAgro 2019}

diferentes florestas tropicais é dificultada por fatores intrínsecos (altura e crescimento) e extrínsecos (clima, edafologia e morfologia) que afetam o crescimento individual das árvores, resultando em variações, tais como: a competição de espécies, o grau de perturbação e o período de tempo desde a época em que ocorreu a perturbação (SILVA, 1989).

Carvalho (2000) classifica maçaranduba, como espécie tolerante à sombra, mas a luz acelera o seu crescimento. Korning; Balslev (1994), avaliando 22 espécies arbóreas em floresta tropical na Amazônia (Equador), identificaram cinco espécies com estratégia semelhante à apresentada pela maçaranduba. Tais espécies eram preferenciais de dossel ou subdossel, tolerantes à sombra, mas respondiam oportunisticamente, em termos de crescimento, a melhorias nas condições luminosas.

\section{Conclusões}

A maçaranduba está entre as principais espécies florestais exportadas pelo estado do Pará, na forma de madeira serrada, com mercado seguro nos Estados Unidos da América, Japão, e em vários países europeus. Essa espécie pertence ao grupo ecológico das tolerantes a sombra, no entanto, essas espécies geralmente germinam sob o dossel, sua vida reprodutiva nessa situação fica aguardando a oportunidade para atingir o dossel superior para só então reproduzir. Devido ao efeito da abertura do dossel está ligada diretamente no crescimento das árvores, estas diminuem a competição por espaço e luz aumentando com o passar do tempo, provocando a redução do crescimento populacional da maçaranduba, no caso dessa espécie o ciclo de corte deve ser maior pois a mesma não se recuperou em 27 anos de estudos. Portanto devemos planejar o manejo florestal executando a exploração de forma sustentável possibilitando a conservação do ecossistema e fazendo com que a produção de madeira tenha um rendimento maior, por essa razão é importante conhecer o crescimento das espécies de interesse econômico. 


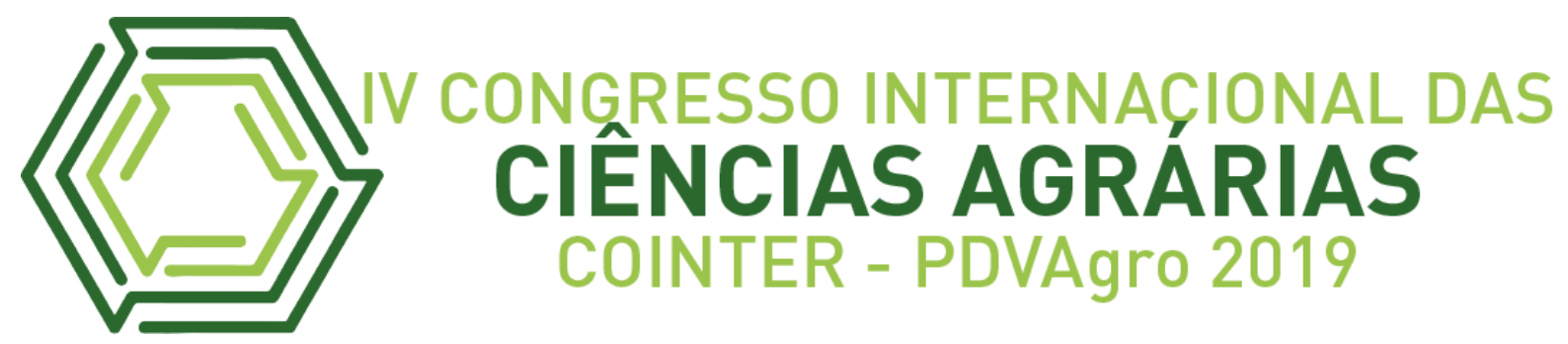

\section{Referências}

ASSOCIAÇÃ0 DAS INDUSTRIAS EXPORTADORAS DE MADEIRAS DO ESTADO DO PARA. Exportação brasileira de madeira. Belém: SECEX/DECEX, 2005. 4p.

AZEVEDO, C. P. de; SANQUETTA, C. R.; SILVA, J. N. M.; MACHADO, S. do A. Efeito da exploração de madeira e dos tratamentos Silviculturais no agrupamento ecológico de espécies. FLORESTA, Curitiba, PR, v. 38, n. 1, jan./mar. 2008.

BARROS, A. V. de; BARROS, P. L. C. de; SILVA, L. C. B. da. Estudo da diversidade de espécies de uma floresta situado em Curuá-Una - Pará. Revista de Ciências Agrárias, Belém, n. 34, p. 49-65, jan./jun. 2000.

CARVALHO, J. O. P. Classificação em grupos ecológicos das espécies mais importantes em uma área de Floresta Nacional do Tapajós, Belterra, PA. Belém: Embrapa Amazônia Oriental, 2000. 4 p. (Embrapa Amazônia Oriental. Comunicado Técnico, 41).

CARVALHO, J. O. P. Estrutura de Matas altas sem babaçu na Floresta Nacional do Tapajós. In: SILVA, J. N. M.; CARVALHO, J. O. P.; YARED, J. A. G.A Silvicultura na Amazônia Oriental: contribuições do projeto Embrapa/DFID. Belém: Embrapa Amazônia Oriental. DFID. 2001. p. 277290.

CHIPAIA, F. C.; REIS, A. R. S.; REIS, L. P.; CARVALHO, J. C.; SILVA, E. F. R. Description anatomical macroscopic wood forest species of eight market in the municipality of Altamira-PA. J. Bioen. Food Sci., v. 2, n.1: p.18-24, 2015.

FRANCEZ, L. M. de B.; CARVALHO, J. O. P. de; JARDIM, F. C. da S.; QUANZ, B.; PINHEIRO, K. A. O. Efeito de duas intensidades de colheitas de madeira na estrutura de uma floresta natural na região de Paragominas, PA. Revista Acta Amazônica, Manaus, v. 39, n. 4, p. 851 - 864. 2009.

GAYOT, M.; SIST, P. Vulnerabilite des especes de maçaranduba face a l'exploitation en Amazonie bresilienne: nouvelles normes d'exploitation a \& fink. Bois et Forets des Tropiques: Forets Amazoniennes, v.2, n.280, p. 75-90, 2004.

GOMES, J. I.; SILVA E. M. A.; MELO, A. T. S. Durabilidade de 15 espécies de madeiras amazônica em contato com o solo em ambiente sombreado. Belém: Embrapa Amazônia Oriental, 2005. 4 p. (Comunicado Técnico, 148).

IDEFLOR. Instituto de desenvolvimento Florestal do Estado do Pará. Plano Anual de Outorga Florestal de 2009. Belém: IDEFLOR, 2008. 68p.

JARDIM, F. C. da S.; HOSOKAWA, R. T. Estrutura da floresta equatorial úmida da estação experimental de silvicultura tropical do INPA. Acta Amazônica, Manaus, v. 16/17, p. 411-508. 1986/1987. 


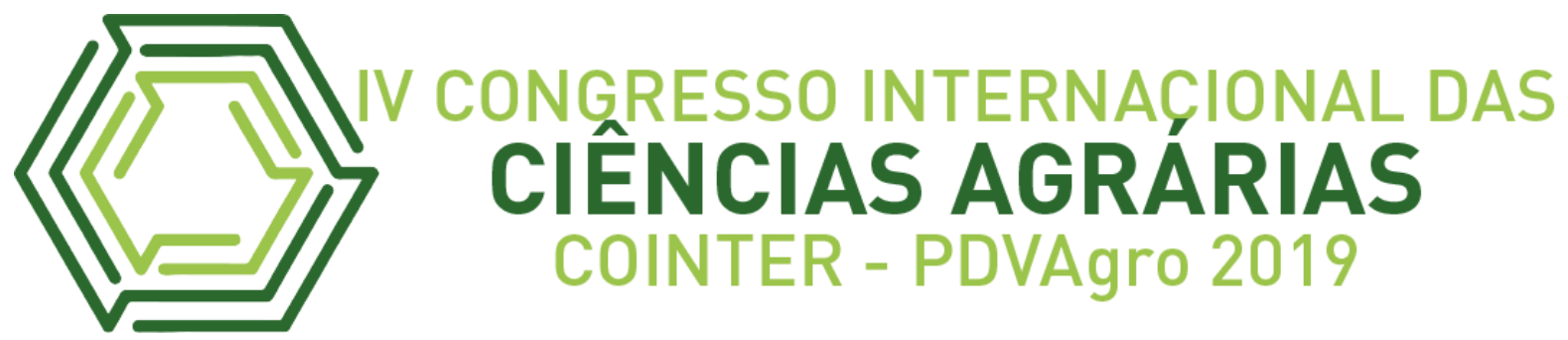

KORNING, J.; BALSLEV, H. Growth rates and mortality patterns of tropical lowland tree species and the relation to forest structure in Amazonian Ecuador. Journal of Tropical Ecology, v.10, p 151166, 1994.

LOUREIRO, A. A.; SILVA, M. F. Catalogo das madeiras da Amazônia. Belém: SUDAM, 1968. p.167-168.

LOUREIRO, A. A. Essências madeireiras da Amazônia. Manaus: INPA, 1979. 2 v.

MARTINELLI, G.; MORAES, M. Á. Livro vermelho da flora do Brasil / texto e organização Gustavo Martinelli, Miguel Ávila Moraes; tradução Flavia Anderson, Chris Hieatt. - 1. ed. - Rio de Janeiro: Andrea Jakobsson: Instituto de Pesquisas Jardim Botânico do Rio de Janeiro, 2013. 1100 p.; $30 \mathrm{~cm}$.

MIRANDA, I. S. Análise florística e estrutural da vegetação lenhosa do rio Comemoração, Pimenta Bueno, Rondônia, Brasil. Revista Acta Amazônica. v. 30, n. 3, p. 393-422. 2000.

OLIVEIRA, L.C. de. Dinâmica de crescimento e regeneração natural de uma floresta secundária no Estado do Pará. 1995. (Mestrado) - UFPA, Belém, 1995.

SABOGAL, C.; LENTINE, M.; POKORNY, B.; SILVA, J. M. N.; ZWEEDE, J.; VERÍSSIMO. A.; BOSCOLO. M. Manejo empresarial na Amazônia brasileira. Belém: CIFOR. 174 p. 2006.

SILVA, J. N. M.; LOPES, J. do C.A. Inventário florestal contínuo em florestas tropicais: a metodologia da EMBRAPA-CPATU na Amazônia brasileira. Belém: EMBRAPA-CPATU (EMBRAPA-CPATU. Documentos, 33), 1984. 36 p.

SILVA, J. N. M.; SILVA, S. M. A. da; COSTA, D. H. M.; BAIMA. A. M. V.; OLIVEIRA, L. C. de; CARVALHO, J. O. P.; LOPES, J. do C. A. Crescimento, mortalidade e recrutamento em florestas de terra firme da Amazônia Oriental: observações nas regiões do Tapajós e Jari. In: SILVA, J. N. M.; CARVALHO, J. O. P.; YARED, J. A. G. A silvicultura na Amazônia Oriental: contribuições do projeto Embrapa/DFID. Belém: Embrapa Amazônia Oriental: DFID. p. 291-308, 2001.

SILVA, J. N. M.; LOPES, J. do C. A.; OLIVEIRA, L. C. de; SILVA, S. M. A. da; CARVALHO, J. O. P. de; COSTA, D. H. M.; MELO, M. S.; TAVARES, M. J. M. Diretrizes para Instalação e Medição de Parcelas Permanentes em Florestas Naturais da Amazônia Brasileira. Belém: Embrapa / ITTO. 68p. 2005.

SOUZA, M. H.; MAGLIANO, M. M.; CAMARGO, J. A. A.; SOUZA, M. R. Madeiras tropicais brasileiras. 2. ed. Belém: 1BAMALaboratorio de Produtos Florestais, 2002. p. 82- 83.

SUPERINTENDÊNCIA DE DESENVOLVIMENTO DA AMAZÔNIA. Departamento de Recursos Naturais Centro de Tecnologia Madeira. Pesquisa e Informações sobre espécies. Belém, 1979.

SCHULZE, M.; VIDAL, E.; GROGAN, J.; ZWEEDE, J.; ZARIN, D. Madeiras nobres em perigo. Ciência Hoje. v. 36, n.214, p. 66-69, 2005. 


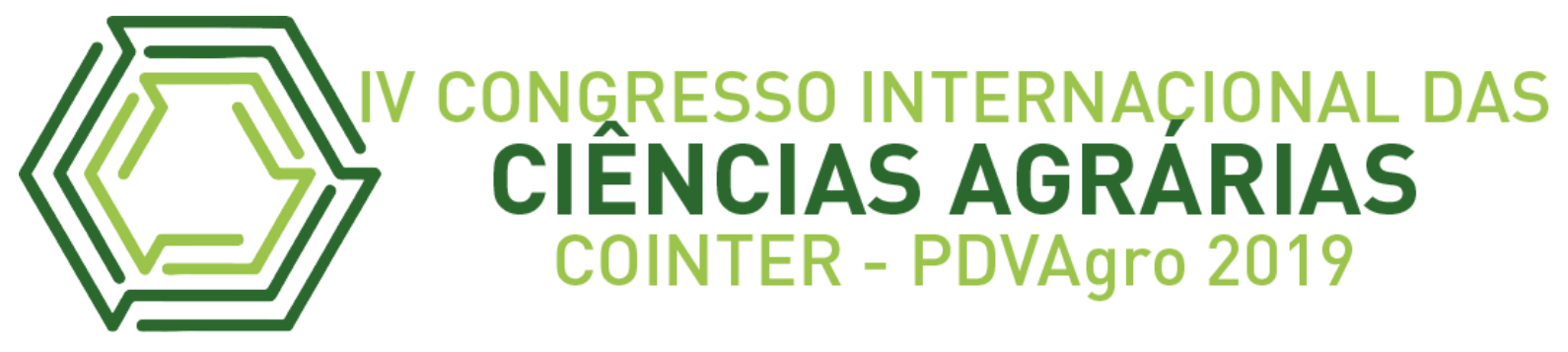

TAKEDA, P. S.; GURGEL, E. S. C. CARVALHO, A. C. M.; SANTOS, J. U. M. dos. Manilkara huberi Standley (Sapotaceae: aspectos morfológicos do fruto, da semente e do tipo de geminação. In: CONGRESSO NACIONAL DE BOTÂNICA, 54, 2003. Anais... Belém: MPEG/SBB, 2003.

WHITMORE, T.C. An introduction to tropical rain forests. Oxford: Oxford University Press, 1990. p. 99-132. 


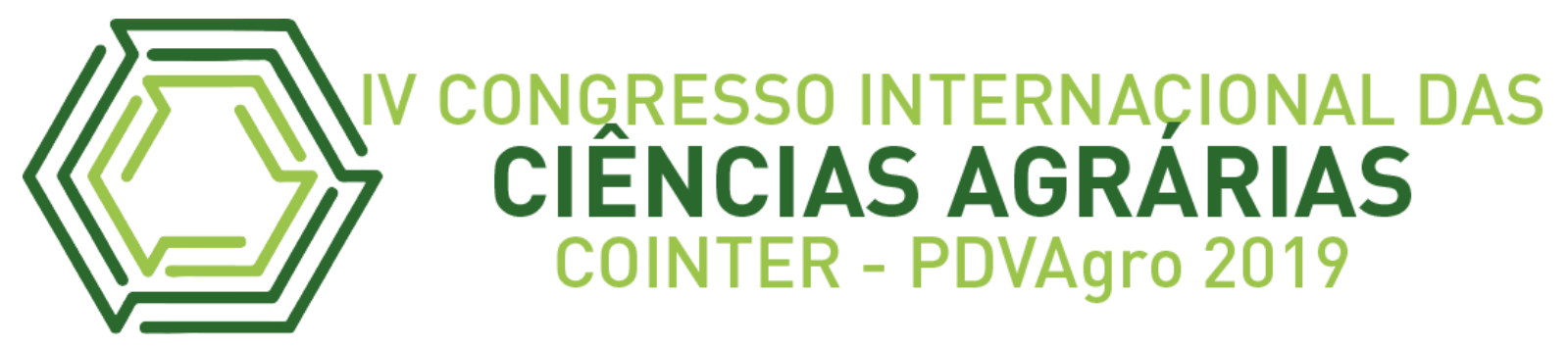

